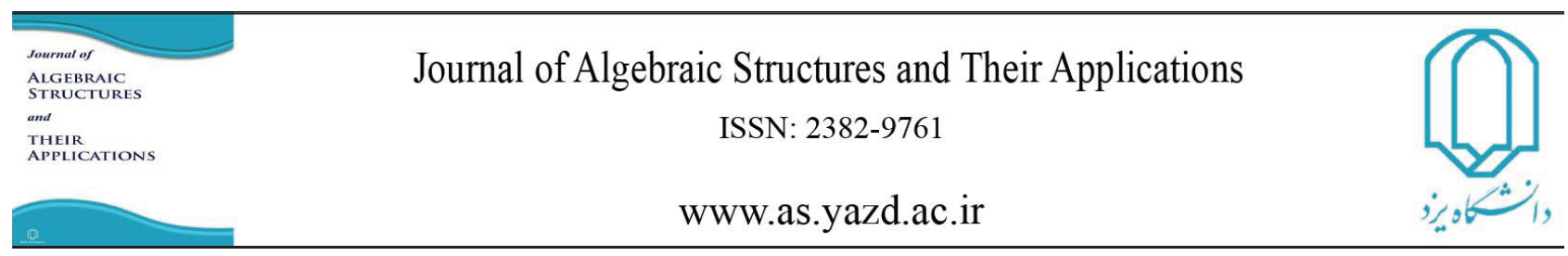

Algebraic Structures and Their Applications Vol. 4 No. 1 ( 2017 ) pp 59-78.

\title{
A NOTE ON A GRAPH RELATED TO THE COMAXIMAL IDEAL GRAPH OF A COMMUTATIVE RING
}

\author{
SUBRAMANIAN VISWESWARAN* AND JAYDEEP PAREJIYA
}

Communicated by S. Alikhani

\begin{abstract}
The rings considered in this article are commutative with identity which admit at least two maximal ideals. This article is inspired by the work done on the comaximal ideal graph of a commutative ring. Let $R$ be a ring. We associate an undirected graph to $R$ denoted by $\mathcal{G}(R)$, whose vertex set is the set of all proper ideals $I$ of $R$ such that $I \nsubseteq J(R)$, where $J(R)$ is the Jacobson radical of $R$ and distinct vertices $I_{1}, I_{2}$ are adjacent in $\mathcal{G}(R)$ if and only if $I_{1} \cap I_{2}=I_{1} I_{2}$. The aim of this article is to study the interplay between the graph-theoretic properties of $\mathcal{G}(R)$ and the ring-theoretic properties of $R$.
\end{abstract}

\section{INTRODUCTION}

The rings considered in this article are commutative with identity which admit at least two maximal ideals. Let $R$ be a ring. We denote the set of all maximal ideals of $R$ by $\operatorname{Max}(R)$. This article is inspired by the interesting theorems proved by M. Ye and T. Wu in [15]. Motivated http://dx.doi.org/10.29252/asta.4.1.57

MSC(2010): Primary 13A15; Secondary 05C25.

Keywords: Comaximal ideal graph of a commutative ring, complete graph, von Neumann regular ring, bipartite graph, clique number.

Received: 11 November 2017, Accepted: 19 April 2018

* Corresponding author 
by the research work done on the comaximal graph of a ring in [9, ㅍ, ㅍ, ㅍ.3, 14] and on the annihilating-ideal graph of a ring in [5, 6], M. Ye and T. Wu in [15] introduced and studied a graph structure on a ring $R$ whose vertex set is the set of all proper ideals $I$ of $R$ such that $I \nsubseteq J(R)$, where $J(R)$ is the Jacobson radical of $R$ and distinct vertices $I_{1}$ and $I_{2}$ are adjacent if and only if $I_{1}+I_{2}=R$. M. Ye and T. Wu called the graph introduced and investigated by them in [15] as the comaximal ideal graph of $R$ and denoted it by $\mathscr{C}(R)$ and they investigated the influence of certain graph parameters of $\mathscr{C}(R)$ on the ring structure of $R$.

We denote the cardinality of a set $A$ by $|A|$. Let $R$ be a ring with $|\operatorname{Max}(R)| \geq 2$. In this article, we introduce a graph structure on $R$, denoted by $\mathcal{G}(R)$, is an undirected graph whose vertex set is the set of all proper ideals $I$ of $R$ such that $I \nsubseteq J(R)$ and distinct vertices $I_{1}, I_{2}$ are adjacent in $\mathcal{G}(R)$ if and only if $I_{1} \cap I_{2}=I_{1} I_{2}$. The graphs considered in this article are undirected and simple. We denote the set of all vertices of a graph $G$ by $V(G)$ and the set of all edges of $G$ by $E(G)$. A subgraph $H$ of a graph $G$ is said to be a spanning subgraph of $G$ if $V(G)=V(H)$. Let $R$ be a ring. If $I_{1}, I_{2}$ are ideals of $R$ such that $I_{1}+I_{2}=R$, then we know from [2, Proposition $1.10(i)$ ] that $I_{1} \cap I_{2}=I_{1} I_{2}$. Let $I_{1}, I_{2}$ be proper ideals of a ring such that $I_{i} \not \subset J(R)$ for each $i \in\{1,2\}$. If $I_{1}$ and $I_{2}$ are adjacent in $\mathscr{C}(R)$, then they are adjacent in $\mathcal{G}(R)$. This shows that $\mathscr{C}(R)$ is a spanning subgraph of $\mathcal{G}(R)$. Hence, it is natural to compare the graph-theoretic properties of $\mathcal{G}(R)$ with that of the graph-theoretic properties of $\mathscr{C}(R)$. The aim of this article is to study the interplay between the graph-theoretic properties of $\mathcal{G}(R)$ and the ring-theoretic properties of $R$.

First, it is useful to recall certain definitions and results from commutative ring theory that are used in this article. Let $R$ be a ring. The nil radical of $R$ is denoted by $\operatorname{nil}(R)$. A ring $R$ is said to be reduced if $\operatorname{nil}(R)=(0)$. Recall from [G, Exercise 16, page 111] that a ring $R$ is said to be von Neumann regular if for each $a \in R$, there exists $b \in R$ such that $a=a^{2} b$. A principal ideal ring is said to be a special principal ideal ring (SPIR) if $R$ has a unique prime ideal. If $\mathfrak{m}$ is the only prime ideal of a special principal ideal ring $R$, then we denote it by mentioning that $(R, \mathfrak{m})$ is a SPIR. If $(R, \mathfrak{m})$ is a $\operatorname{SPIR}$, then $\mathfrak{m}$ is nilpotent. Let $(R, \mathfrak{m})$ be a SPIR which is not a field. Let $n \geq 2$ be least with the property that $\mathfrak{m}^{n}=(0)$. Then it follows from the proof of $(i i i) \Rightarrow(i)$ of $\left[2\right.$, Proposition 8.8] that $\left\{\mathfrak{m}^{i} \mid i \in\{1, \ldots, n-1\}\right\}$ is the set of all nonzero proper ideals of $R$. A ring with a unique maximal ideal is referred to as a quasilocal ring. A ring which admits only a finite number of maximal ideals is referred to as a semiquasilocal ring. A Noetherian quasilocal (respectively, semiquasilocal) ring is referred to as a local (respectively, semilocal) ring. For a ring $R$, we denote the set of all units of $R$ by $U(R)$ and the set of all nonunits of $R$ by $N U(R)$. The Krull dimension of a ring $R$ is simply denoted by $\operatorname{dim} R$. We use $\operatorname{Spec}(R)$ to denote the set of all prime ideals of a ring $R$. We use $\subset$ to denote proper inclusion. For any $n \geq 2$, we denote the ring of integers modulo $n$ by $\mathbb{Z}_{n}$. 
Next, it is useful to recall the following results from graph theory before we give an account of the results that are proved in this article. Let $G=(V, E)$ be a graph. Let $a, b \in V, a \neq b$. Recall that the distance between $a$ and $b$, denoted by $d(a, b)$ is defined as the length of a shortest path in $G$ between $a$ and $b$ if there exists such a path in $G$; otherwise, we define $d(a, b)=\infty$. We define $d(a, a)=0$. The diameter of $G$, denoted by $\operatorname{diam}(G)$, is defined as $\operatorname{diam}(G)=\sup \{d(a, b) \mid a, b \in V\}[3]$. A graph $G=(V, E)$ is said to be connected if for any distinct $a, b \in V$, there exists a path in $G$ between $a$ and $b[3]$. Let $G=(V, E)$ be a connected graph. Let $a \in V$. Then the eccentricity of $a$, denoted by $e(a)$, is defined as $e(a)=$ $\sup \{d(a, b) \mid b \in V\}$. The radius of $G$, denoted by $r(G)$, is defined as $r(G)=\min \{e(a) \mid a \in V\}$. A simple graph $G=(V, E)$ is said to be complete if every pair of distinct vertices of $G$ are adjacent in $G$ [3, Definition 1.1.11]. Let $n \in \mathbb{N}$. A complete graph on $n$ vertices is denoted by $K_{n}$. A graph $G=(V, E)$ is said to be bipartite if $V$ can be partitioned into two noempty subsets $V_{1}$ and $V_{2}$ such that each edge of $G$ has one end in $V_{1}$ and the other in $V_{2}$. A bipartite graph with vertex partition $V_{1}$ and $V_{2}$ is said to be complete if each element of $V_{1}$ is adjacent to every element of $V_{2}$.

Let $G=(V, E)$ be a graph. Recall from [3, Definition 1.2.2] that a clique of $G$ is a complete subgraph of $G$. The clique number of $G$, denoted by $\omega(G)$, is defined as the largest integer $n \geq 1$ such that $G$ contains a clique on $n$ vertices [3, page 185]. We set $\omega(G)=\infty$ if $G$ contains a clique on $n$ vertices for all $n \geq 1$. Recall from [3, page 129] that a vertex coloring of $G$ is a map $f: V \rightarrow S$, where $S$ is a set of distinct colors. A vertex coloring $f: V \rightarrow S$ is said to be proper, if adjacent vertices of $G$ receive different colors of $S$; that is, if $a$ and $b$ are adjacent vertices of $G$, then $f(a) \neq f(b)$. The chromatic number of $G$, denoted by $\chi(G)$, is the minimum number of colors needed for a proper vertex coloring of $G$ [3, Definition 7.1.2]. It is well-known that for any graph $G, \omega(G) \leq \chi(G)$.

Let $R$ be a ring with $|\operatorname{Max}(R)| \geq 2$. It is shown in Section $\square$ of this article that $\mathcal{G}(R)$ is connected and $\operatorname{diam}(\mathcal{G}(R)) \leq 3$. With the hypothesis that $J(R)=(0)$, it is shown that $\mathcal{G}(R)$ is complete if and only if $R$ is von Neumann regular. Some classes of rings $R$ are provided such that $\operatorname{diam}(\mathcal{G}(R))$ is either 2 or 3 . Moreover, some examples are given to illustrate the results proved in this section.

Let $R$ be a ring with $|\operatorname{Max}(R)| \geq 2$. It is proved in Section 3 of this article that $\mathcal{G}(R)$ is a finite bipartite graph if and only if $R \cong F_{1} \times F_{2}$ as rings, where $F_{i}$ is a field for each $i \in\{1,2\}$.

Let $R$ be a ring such that $|\operatorname{Max}(R)| \geq 2$. With the assumption that $J(R)=(0)$, it is proved in Section $\mathbb{\theta}$ of this article that $\omega(\mathcal{G}(R))<\infty$ if and only if there exist $n \geq 2$ and fields $F_{1}, F_{2}, \ldots, F_{n}$ such that $R \cong F_{1} \times F_{2} \times \cdots \times F_{n}$ as rings. Moreover, in such a case, it is verified that $\omega(\mathcal{G}(R))=\chi(\mathcal{G}(R))=2^{n}-2$. Moreover, an example of a ring $R$ with $|\operatorname{Max}(R)|=2$ is provided such that $\omega(\mathcal{G}(R))=5<\chi(\mathcal{G}(R))=6$. 


\section{Some basic properties of $\mathcal{G}(R)$}

Let $R$ be a ring such that $|\operatorname{Max}(R)| \geq 2$. The aim of this section is to study some basic properties of $\mathcal{G}(R)$.

Proposition 2.1. Let $R$ be a ring such that $|\operatorname{Max}(R)| \geq 2$. Then $\mathcal{G}(R)$ is connected with $\operatorname{diam}(\mathcal{G}(R)) \leq 3$.

Proof. It is already noted in the introduction that $\mathscr{C}(R)$ is a spanning subgraph of $\mathcal{G}(R)$. We know from [15, Theorem 2.4] that $\mathscr{C}(R)$ is connected with $\operatorname{diam}(\mathscr{C}(R)) \leq 3$. Therefore, $\mathcal{G}(R)$ is connected with $\operatorname{diam}(\mathcal{G}(R)) \leq 3$.

Proposition 2.2. Let $R$ be a ring such that $|\operatorname{Max}(R)| \geq 2$. If either $J(R)=(0)$ or $J(R)$ is a prime ideal of $R$, then $\operatorname{diam}(\mathcal{G}(R)) \leq 2$.

Proof. Let $I_{1}, I_{2} \in V(\mathcal{G}(R))$ be such that $I_{1} \neq I_{2}$. We claim that $d\left(I_{1}, I_{2}\right) \leq 2$ in $\mathcal{G}(R)$. This is clear if $I_{1}, I_{2}$ are adjacent in $\mathcal{G}(R)$. Hence, we can assume that $I_{1}$ and $I_{2}$ are not adjacent in $\mathcal{G}(R)$. We claim that $I_{1} \cap I_{2} \not \subset J(R)$. We are assuming that either $J(R)=(0)$ or $J(R)$ is a prime ideal of $R$. We consider the following cases.

$\operatorname{Case}(i) J(R)=(0)$

Observe that $I_{1} \cap I_{2} \neq(0)$. For if $I_{1} \cap I_{2}=(0)$, then $I_{1} \cap I_{2}=I_{1} I_{2}=(0)$ and this implies that $I_{1}$ and $I_{2}$ are adjacent in $\mathcal{G}(R)$. This is in contradiction to the assumption that $I_{1}$ and $I_{2}$ are not adjacent in $\mathcal{G}(R)$. Therefore, $I_{1} \cap I_{2} \neq(0)$. As $J(R)=(0)$, we get that $I_{1} \cap I_{2} \nsubseteq J(R)$. Case $(i i) J(R)$ is a prime ideal of $R$

Let $i \in\{1,2\}$. As $I_{i} \in V(\mathcal{G}(R))$, we obtain that $I_{i} \nsubseteq J(R)$. Since $J(R) \in \operatorname{Spec}(R)$, we obtain from [2, Proposition 1.11(ii)] that $I_{1} \cap I_{2} \not \subset J(R)$.

Thus $I_{1} \cap I_{2} \nsubseteq J(R)$. Hence, there exists $\mathfrak{m} \in \operatorname{Max}(R)$ such that $I_{1} \cap I_{2} \nsubseteq \mathfrak{m}$. This implies that $I_{i} \not \mathbb{m}$ for each $i \in\{1,2\}$. Therefore, $I_{i}+\mathfrak{m}=R$ for each $i \in\{1,2\}$. Hence, $I_{1}-\mathfrak{m}-I_{2}$ is a path of length two in $\mathscr{C}(R)$ and this is also a path in $\mathcal{G}(R)$ since $\mathscr{C}(R)$ is a spanning subgraph of $\mathcal{G}(R)$. This proves that $d\left(I_{1}, I_{2}\right) \leq 2$ in $\mathcal{G}(R)$ for any $I_{1}, I_{2} \in V(\mathcal{G}(R))$. Therefore, $\operatorname{diam}(\mathcal{G}(R)) \leq 2$.

Remark 2.3. (i) Let $R$ be a ring such that $|\operatorname{Max}(R)| \geq 2$. If $J(R) \in \operatorname{Spec}(R)$, then $\operatorname{diam}(\mathscr{C}(R)) \leq 2$.

(ii) Let $R$ be a ring such that $|\operatorname{Max}(R)| \geq 3$. Suppose that there exist $I_{1}, I_{2} \in V(\mathscr{C}(R))$ such that $I_{1}$ and $I_{2}$ are not adjacent in $\mathscr{C}(R)$ and $I_{1} I_{2} \subseteq J(R)$. Then $\operatorname{diam}(\mathscr{C}(R))=3$.

Proof. $(i)$ Let $I_{1}, I_{2} \in V(\mathscr{C}(R))$ be such that $I_{1} \neq I_{2}$ and $I_{1}, I_{2}$ are not adjacent in $\mathscr{C}(R)$. Using the hypothesis that $J(R) \in \operatorname{Spec}(R)$, it is noted in the proof of Case $(i i)$ of Proposition 
2.2 that there exists $\mathfrak{m} \in \operatorname{Max}(R)$ such that $I_{1}-\mathfrak{m}-I_{2}$ is a path of length two between $I_{1}$ and $I_{2}$ in $\mathscr{C}(R)$. Hence, $\operatorname{diam}(\mathscr{C}(R)) \leq 2$.

(ii) Now, by assumption $I_{1} \cdot I_{2} \in V(\mathscr{C}(R))$ are such that $I_{1}, I_{2}$ are not adjacent in $\mathscr{C}(R)$ and $I_{1} I_{2} \subseteq J(R)$. Since $I_{1} \nsubseteq J(R), I_{1} I_{2} \subseteq J(R)$, and $J(R)$ is a radical ideal of $R$, it follows that $I_{1} \neq I_{2}$. We claim that $d\left(I_{1}, I_{2}\right)=3$ in $\mathscr{C}(R)$. We know from [15, Theorem 2.4] that $\mathscr{C}(R)$ is connected and $\operatorname{diam}(\mathscr{C}(R)) \leq 3$. We verify that there exists no path of length two between $I_{1}$ and $I_{2} \in \mathscr{C}(R)$. Suppose that there exists a path of length two between $I_{1}$ and $I_{2}$ in $\mathscr{C}(R)$. Thus there exists $I \in V(\mathscr{C}(R))$ such that $I_{1}-I-I_{2}$ is a path of length two in $\mathscr{C}(R)$. Hence, $I_{i}+I=R$ for each $i \in\{1,2\}$. Let $\mathfrak{m} \in \operatorname{Max}(R)$ be such that $I \subseteq \mathfrak{m}$. Note that $I_{i}+\mathfrak{m}=R$ for each $i \in\{1,2\}$. As $I_{1} I_{2} \subseteq J(R) \subset \mathfrak{m}$, we get that either $I_{1} \subseteq \mathfrak{m}$ or $I_{2} \subseteq \mathfrak{m}$. Therefore, $I_{i}+\mathfrak{m} \neq R$ for at least one $i \in\{1,2\}$. This is a contradiction. Therefore, $d\left(I_{1}, I_{2}\right) \geq 3$ in $\mathscr{C}(R)$ and so, we obtain that $\operatorname{diam}(\mathscr{C}(R))=3$.

Let $R$ be a ring such that $|\operatorname{Max}(R)| \geq 2$. Suppose that $J(R)=(0)$. In Theorem 2.4 , we characterize rings $R$ such that $\mathcal{G}(R)$ is complete.

Theorem 2.4. Let $R$ be a ring with $|\operatorname{Max}(R)| \geq 2$. Suppose that $J(R)=(0)$. Then the following statements are equivalent:

(i) $\mathcal{G}(R)$ is complete.

(ii) $R$ is von Neumann regular.

Proof. $(i) \Rightarrow(i i)$ Assume that $\mathcal{G}(R)$ is complete. By hypothesis, $J(R)=(0)$. Since $n i l(R) \subseteq$ $J(R)$, we obtain that $\operatorname{nil}(R)=(0)$. Hence, $R$ is reduced. Therefore, to prove $R$ is von Neumann regular, it follows from $(d) \Rightarrow(a)$ of [ [7, Exercise 16, page 111] that it is enough to show that $\operatorname{dim} R=0$. Let $\mathfrak{p} \in \operatorname{Spec}(R)$. Let $a \in R \backslash \mathfrak{p}$. We claim that $\mathfrak{p}+R a=R$. If $R a=R a^{2}$, then $a=r a^{2}$ for some $r \in R$. Hence, $a(1-r a)=0 \in \mathfrak{p}$. As $a \notin \mathfrak{p}$, we obtain that $1-r a \in \mathfrak{p}$. This implies that $\mathfrak{p}+R a=R$. Suppose that $R a \neq R a^{2}$. Then $R a, R a^{2} \in V(\mathcal{G}(R))$. Since $\mathcal{G}(R)$ is complete, we obtain that $R a$ and $R a^{2}$ are adjacent in $\mathcal{G}(R)$. Therefore, $R a \cap R a^{2}=R a^{3}$. Thus $R a^{2}=R a^{3}$ and this implies that $a^{2}=s a^{3}$ for some $s \in R$. Hence, $a^{2}(1-s a)=0$. From $a^{2} \notin \mathfrak{p}$, we get that $1-s a \in \mathfrak{p}$. Therefore, $\mathfrak{p}+R a=R$. This proves that $\mathfrak{p}$ is a maximal ideal of $R$ for any $\mathfrak{p} \in \operatorname{Spec}(R)$. Therefore, $\operatorname{dim} R=0$ and so, $R$ is von Neumann regular.

$($ ii $) \Rightarrow(i)$ We are assuming that $R$ is von Neumann regular. Let $a \in R$. We know from $(1) \Rightarrow(3)$ of $[7$, Exercise 29, page 113] that there exist a unit $u \in R$ and an idempotent element $e$ of $R$ such that $a=u e$. Using this fact, it can be shown that any ideal of $R$ is a radical ideal of $R$. Let $I_{1}, I_{2} \in V(\mathcal{G}(R))$ with $I_{1} \neq I_{2}$. We know from [2, Exercise 1.13(iii), page 9] that $\sqrt{I_{1} I_{2}}=\sqrt{I_{1} \cap I_{2}}$. Since for any ideal $I$ of $R, I=\sqrt{I}$, we obtain that $I_{1} \cap I_{2}=I_{1} I_{2}$. Hence, $I_{1}$ and $I_{2}$ are adjacent in $\mathcal{G}(R)$. This proves that $\mathcal{G}(R)$ is complete. 
Example 2.5. Let $F_{n}$ be a field for each $n \in \mathbb{N}$. Let $R=\prod_{n=1}^{\infty} F_{n}$. Then $\mathcal{G}(R)$ is complete but $\operatorname{diam}(\mathscr{C}(R))=3$.

Proof. Note that $R$ is von Neumann regular. We know from $(a) \Rightarrow(d)$ of [ [ $]$, Exercise 16, page 111 ] that $R$ is reduced and $\operatorname{dim} R=0$. Hence, $J(R)=\operatorname{nil}(R)=(0)$. It now follows from $(i i) \Rightarrow$ $(i)$ of Theorem [2.4 that $\mathcal{G}(R)$ is complete. We next verify that $\operatorname{diam}(\mathscr{C}(R))=3$. We know from [15, Theorem 2.4] that $\mathscr{C}(R)$ is connected and $\operatorname{diam}(\mathscr{C}(R)) \leq 3$. Note that $\operatorname{Max}(R)$ is infinite and $J(R)=(0,0,0, \ldots$,$) . Let I_{1}=\left\{\left(\alpha_{1}, \alpha_{2}, \alpha_{3}, \ldots,\right) \in R \mid \alpha_{i}=0\right.$ for all $i \in \mathbb{N}$ with $i$ odd $\}$ and let $I_{2}=\left\{\left(0, \alpha_{2}, \alpha_{3}, \alpha_{4} \ldots,\right) \in R \mid \alpha_{i}=0\right.$ for all $i \in \mathbb{N}$ with $i$ even $\}$. Observe that $I_{1}, I_{2} \in$ $V(\mathscr{C}(R))$ with $I_{1} \neq I_{2}$ and $I_{1}+I_{2} \neq R$. Therefore, $I_{1}$ and $I_{2}$ are not adjacent in $\mathscr{C}(R)$. As $I_{1} I_{2}=(0,0,0, \ldots,) \subseteq J(R)$, we obtain from the proof of Remark [2.3(ii) that $d\left(I_{1}, I_{2}\right) \geq 3$ in $\mathscr{C}(R)$ and so, it follows that $\operatorname{diam}(\mathscr{C}(R))=3$.

Corollary 2.6. Let $R$ be a ring such that $|\operatorname{Max}(R)| \geq 2$. Suppose that $J(R)=(0)$. If $R$ is not von Neumann regular, then $\operatorname{diam}(\mathcal{G}(R))=2$.

Proof. Let $\mathfrak{m} \in \operatorname{Max}(R)$. As $|\operatorname{Max}(R)| \geq 2$, it follows that $\mathfrak{m} \in V(\mathcal{G}(R))$. Let $\mathfrak{m}, \mathfrak{m}^{\prime} \in \operatorname{Max}(R)$ be such that $\mathfrak{m} \neq \mathfrak{m}^{\prime}$. As $\mathfrak{m}+\mathfrak{m}^{\prime}=R$, we obtain that $\mathfrak{m}$ and $\mathfrak{m}^{\prime}$ are adjacent in $\mathscr{C}(R)$ and so, they are adjacent in $\mathcal{G}(R)$. Hence, $\mathcal{G}(R)$ admits at least one edge. We know from Proposition $[1$ that $\mathcal{G}(R)$ is connected. We are assuming that $J(R)=(0)$ and $R$ is not von Neumann regular. Therefore, we obtain from $(i) \Rightarrow(i i)$ of Theorem 2.4 that $\operatorname{diam}(\mathcal{G}(R)) \geq 2$. Since $J(R)=(0)$, we know from Proposition 2.2 that $\operatorname{diam}(\mathcal{G}(R)) \leq 2$. Therefore, we get that $\operatorname{diam}(\mathcal{G}(R))=2$.

Note that $J(\mathbb{Z})=(0)$ and $\mathbb{Z}$ is not von Neumann regular. Hence, we obtain from Corollary 2. that $\operatorname{diam}(\mathcal{G}(\mathbb{Z}))=2$. Let $R$ be a principal ideal domain such that $|\operatorname{Max}(R)| \geq 2$. We verify in Remark 2.7 that $\mathscr{C}(R)=\mathcal{G}(R)$.

Remark 2.7. Let $R$ be a principal ideal domain such that $|\operatorname{Max}(R)| \geq 2$. Then $\mathscr{C}(R)=\mathcal{G}(R)$.

Proof. Let $T$ be any ring with $|\operatorname{Max}(T)| \geq 2$. It is already noted in the introduction that $\mathscr{C}(T)$ is a spanning subgraph of $\mathcal{G}(T)$. Hence, it follows that $\mathscr{C}(R)$ is a spanning subgraph of $\mathcal{G}(R)$. Let $I_{1}, I_{2} \in V(\mathcal{G}(R))$ be such that $I_{1} \neq I_{2}$ and they are adjacent in $\mathcal{G}(R)$. Hence, $I_{1} \cap I_{2}=I_{1} I_{2}$. Since $R$ is a principal domain, there exist nonzero nonunits $a, b \in R$ such that $I_{1}=R a$ and $I_{2}=R b$. As $R$ is a principal ideal domain, it follows that $R a+R b=R d$ and $R a \cap R b=R\left(\frac{a b}{d}\right)$, where $d$ is the greatest common divisor of $a, b$ in $R$. From $I_{1} \cap I_{2}=I_{1} I_{2}$, it follows that $R\left(\frac{a b}{d}\right)=R a b$. This implies that $\frac{a b}{d}=r a b$ for some $r \in R$ and so, $d r=1$. 
Therefore, $d \in U(R)$. Hence, $R a+R b=R d=R$ and so, $I_{1}$ and $I_{2}$ are adjacent in $\mathscr{C}(R)$. This shows that $\mathcal{G}(R)$ is a spanning subgraph of $\mathscr{C}(R)$. Therefore, we obtain that $\mathscr{C}(R)=\mathcal{G}(R)$.

Let $R$ be a ring such that $|\operatorname{Max}(R)| \geq 2$. Suppose that $J(R) \neq(0)$ and $J(R) \in \operatorname{Spec}(R)$. We prove in Corollary 2.10 that $\operatorname{diam}(\mathcal{G}(R))=2$.

Lemma 2.8. Let $R$ be a ring such that $|\operatorname{Max}(R)| \geq 2$. If $\mathcal{G}(R)$ is complete, then $\mathcal{G}\left(\frac{R}{J(R)}\right)$ is complete.

Proof. It is convenient to denote $\frac{R}{J(R)}$ by $T$. Note that $|\operatorname{Max}(T)|=|\operatorname{Max}(R)| \geq 2$ and $J(T)$ is the zero ideal of $T$. Observe that $V(\mathcal{G}(T))$ is the set of all nonzero proper ideals of $T$. Let $A, B \in V(\mathcal{G}(T))$ with $A \neq B$. Note that $A=\frac{I_{1}}{J(R)}$ and $B=\frac{I_{2}}{J(R)}$ for some proper ideals $I_{1}, I_{2}$ of $R$ with $I_{i} \nsubseteq J(R)$ for each $i \in\{1,2\}$. Now, $I_{1}, I_{2} \in V(\mathcal{G}(R))$ with $I_{1} \neq I_{2}$. By hypothesis, $\mathcal{G}(R)$ is complete. Hence, $I_{1}$ and $I_{2}$ are adjacent in $\mathcal{G}(R)$. Therefore, $I_{1} \cap I_{2}=I_{1} I_{2}$. This implies that $\frac{I_{1} \cap I_{2}}{J(R)}=\frac{I_{1} I_{2}}{J(R)}$ and so, $A \cap B=A B$. This shows that $A$ and $B$ are adjacent in $\mathcal{G}(T)$. Therefore, we get that $\mathcal{G}\left(\frac{R}{J(R)}\right)$ is complete.

Corollary 2.9. Let $R$ be a ring such that $|\operatorname{Max}(R)| \geq 2$. If $\mathcal{G}(R)$ is complete, then $\frac{R}{J(R)}$ is von Neumann regular.

Proof. Assume that $\mathcal{G}(R)$ is complete. Let us denote the $\operatorname{ring} \frac{R}{J(R)}$ by $T$. Note that $|\operatorname{Max}(T)|=$ $|\operatorname{Max}(R)| \geq 2$. We know from Lemma 2.8 that $\mathcal{G}(T)$ is complete. Since $J(T)$ is the zero ideal of $T$, we obtain from $(i) \Rightarrow(i i)$ of Theorem $\left[2.4\right.$ that $T=\frac{R}{J(R)}$ is von Neumann regular.

Corollary 2.10. Let $R$ be a ring such that $|\operatorname{Max}(R)| \geq 2$. If $J(R) \in \operatorname{Spec}(R)$, then $\operatorname{diam}(\mathcal{G}(R))=2$.

Proof. It is already noted in the proof of Corollary [2.6] that $\mathcal{G}(R)$ has at least one edge. Let us denote $\frac{R}{J(R)}$ by $T$. Since $J(R) \in \operatorname{Spec}(R)$ by assumption, it follows that $T$ is an integral domain and moreover, we know from Proposition $[2$ that $\operatorname{diam}(\mathcal{G}(R)) \leq 2$. As $|\operatorname{Max}(R)| \geq 2$, it follows that $J(R)$ is not a maximal ideal of $R$. Hence, $T$ is not a field. It is well-known that an integral domain is von Neumann regular if and only if it is a field. Therefore, $T$ is not von Neumann regular and so, it follows from Corollary $[2]$ that $\operatorname{diam}(\mathcal{G}(R)) \geq 2$. Hence, we obtain that $\operatorname{diam}(\mathcal{G}(R))=2$.

Recall from [ [7, page 373] that a ring $R$ is said to be a Hilbert ring if each prime ideal of $R$ is an intersection of maximal ideals of $R$. 
Example 2.11. Let $T=K[X, Y]$ be the polynomial ring in two variables $X, Y$ over a field $K$. Let $I=X^{2} T$ and let $R=\frac{T}{I}$. Then $\operatorname{diam}(\mathcal{G}(R))=2$.

Proof. We know from $(1) \Rightarrow(7)$ of [ $[$, Theorem 31.8] that $T$ is a Hilbert ring and so, we obtain from $(1) \Rightarrow(3)$ of $\left[\right.$ [, Theorem 31.8] that $R=\frac{T}{I}$ is a Hilbert ring. Therefore, $\operatorname{nil}(R)=J(R)$ and as $\operatorname{nil}(R)=\frac{T X}{I}$, we obtain that $J(R)=\frac{T X}{I} \in \operatorname{Spec}(R)$. It is clear that $|\operatorname{Max}(R)| \geq 2$. Indeed, $\operatorname{Max}(R)$ is infinite. Now, it follows from Corollary 2.10 that $\operatorname{diam}(\mathcal{G}(R))=2$.

Remark 2.12. Let $T, I, R$ be as in Example [.]. Since $J(R) \in \operatorname{Spec}(R)$, it follows from $\operatorname{Remark}[2.3](i)$ that $\operatorname{diam}(\mathscr{C}(R)) \leq 2$. Let $I_{1}=\frac{T X}{I}$ and let $I_{2}=\frac{T X^{2}+T Y}{I}$. Note that $I_{1}+I_{2} \neq$ $R$ and so, $I_{1}$ and $I_{2}$ are not adjacent in $\mathscr{C}(R)$. Hence, we obtain that $\operatorname{diam}(\mathscr{C}(R))=2$. Observe that $I_{1} \cap I_{2}=R(X Y+I)=I_{1} I_{2}$. Therefore, $I_{1}$ and $I_{2}$ are adjacent in $\mathcal{G}(R)$ and so, $\mathscr{C}(R) \neq \mathcal{G}(R)$.

We provide an example in Example $[2.5$ to illustrate that Corollary $2.1 \mathrm{~d}$ can fail to hold if the hypothesis that $J(R) \in \operatorname{Spec}(R)$ is omitted.

Lemma 2.13. Let $R$ be a ring such that $|\operatorname{Max}(R)| \geq 2$. Then $e(\mathfrak{m}) \leq 2$ in $\mathscr{C}(R)$ for each $\mathfrak{m} \in \operatorname{Max}(R)$.

Proof. We know from [15, Theorem 2.4] that $\mathscr{C}(R)$ is connected and $\operatorname{diam}(\mathscr{C}(R)) \leq 3$. Let $I \in V(\mathscr{C}(R))$ be such that $I \neq \mathfrak{m}$. If $I \nsubseteq \mathfrak{m}$, then $I+\mathfrak{m}=R$ and so, $I$ and $\mathfrak{m}$ are adjacent in $\mathscr{C}(R)$. Suppose that $I \subset \mathfrak{m}$. Since $I \in V(\mathscr{C}(R))$, there exists $\mathfrak{m}^{\prime} \in \operatorname{Max}(R)$ such that $I \nsubseteq \mathfrak{m}^{\prime}$. It is clear that $I+\mathfrak{m}^{\prime}=\mathfrak{m}+\mathfrak{m}^{\prime}=R$. Hence, $\mathfrak{m}-\mathfrak{m}^{\prime}-I$ is a path of length 2 between $\mathfrak{m}$ and $I$ in $\mathscr{C}(R)$. This proves that $d(\mathfrak{m}, I) \leq 2$ in $\mathscr{C}(R)$ for any $I \in V(\mathscr{C}(R))$. This shows that $e(\mathfrak{m}) \leq 2$ in $\mathscr{C}(R)$ for any $\mathfrak{m} \in \operatorname{Max}(R)$.

Proposition 2.14. Let $R$ be a ring such that $|\operatorname{Max}(R)| \geq 3$. Then $r(\mathscr{C}(R))=2$. If $\operatorname{Max}(R)$ is finite, then $\operatorname{diam}(\mathscr{C}(R))=3$.

Proof. We know from [15, Theorem 2.4] that $\mathscr{C}(R)$ is connected and $\operatorname{diam}(\mathscr{C}(R)) \leq 3$. Let $I \in V(\mathscr{C}(R))$. Then $I$ is a proper ideal of $R$ such that $I \nsubseteq J(R)$. Let $\mathfrak{m}$ be a maximal ideal of $R$ such that $I \subseteq \mathfrak{m}$. We consider the following cases.

$\operatorname{Case}(i) I \neq \mathfrak{m}$

As $I+\mathfrak{m}=\mathfrak{m} \neq R$, it follows that $I$ and $\mathfrak{m}$ are not adjacent in $\mathscr{C}(R)$. Hence, $d(I, \mathfrak{m}) \geq 2$ in $\mathscr{C}(R)$. Therefore, $e(I) \geq 2$ in $\mathscr{C}(R)$.

Case $(i i) I=\mathfrak{m}$ 
Let $\mathfrak{m}^{\prime} \in \operatorname{Max}(R)$ be such that $\mathfrak{m}^{\prime} \neq \mathfrak{m}$. Consider the ideal $\mathfrak{m} \cap \mathfrak{m}^{\prime}$. Since $|\operatorname{Max}(R)| \geq 3$, it is clear that $\mathfrak{m} \cap \mathfrak{m}^{\prime} \nsubseteq J(R)$. As $\mathfrak{m}+\left(\mathfrak{m} \cap \mathfrak{m}^{\prime}\right)=\mathfrak{m} \neq R$, we get that $\mathfrak{m}$ and $\mathfrak{m} \cap \mathfrak{m}^{\prime}$ are not adjacent in $\mathscr{C}(R)$. Hence, $d\left(\mathfrak{m}, \mathfrak{m} \cap \mathfrak{m}^{\prime}\right) \geq 2$ in $\mathscr{C}(R)$. Therefore, $e(\mathfrak{m}) \geq 2$ in $\mathscr{C}(R)$.

This proves that $e(I) \geq 2$ in $\mathscr{C}(R)$ for any $I \in V(\mathscr{C}(R))$. Therefore, $r(\mathscr{C}(R)) \geq 2$.

We know from Lemma 2.13 that $e(\mathfrak{m}) \leq 2$ in $\mathscr{C}(R)$ for each $\mathfrak{m} \in \operatorname{Max}(R)$. Therefore, $e(\mathfrak{m})=2$ in $\mathscr{C}(R)$ for each $\mathfrak{m} \in \operatorname{Max}(R)$. This proves that $r(\mathscr{C}(R))=2$.

Suppose that $|\operatorname{Max}(R)| \geq 3$ and $\operatorname{Max}(R)$ is finite. Let $|\operatorname{Max}(R)|=n$ and let $\left\{\mathfrak{m}_{1}, \mathfrak{m}_{2}, \mathfrak{m}_{3}, \ldots, \mathfrak{m}_{n}\right\}$ denote the set of all maximal ideals of $R$. Let $I_{1}=\mathfrak{m}_{1} \cap \mathfrak{m}_{2}$ and let $I_{2}=\mathfrak{m}_{1} \cap \mathfrak{m}_{3} \cap \cdots \cap \mathfrak{m}_{n}$. Note that $I_{1}, I_{2} \in V(\mathscr{C}(R))$ and $I_{1} \neq I_{2}$. As $I_{1}+I_{2} \subseteq \mathfrak{m}_{1}$, it is clear that $I_{1}$ and $I_{2}$ are not adjacent in $\mathscr{C}(R)$. Observe that $I_{1} I_{2} \subseteq J(R)$. Now, it follows from Remark $\mathbb{L . Z}(i i)$ that $\operatorname{diam}(\mathscr{C}(R))=3$.

Example 2.15. Let $n \geq 3$ and let $p_{1}, p_{2}, p_{3}, \ldots, p_{n}$ be distinct prime numbers. Let $S=$ $\mathbb{Z} \backslash\left(\cup_{i=1}^{n} p_{i} \mathbb{Z}\right)$. Let $R=S^{-1} \mathbb{Z}$. Then $r(\mathcal{G}(R))=2$ and $\operatorname{diam}(\mathcal{G}(R))=3$.

Proof. Note that $R$ is a principal ideal domain and $\operatorname{Max}(R)=\left\{p_{1} R, p_{2} R, p_{3} R, \ldots, p_{n} R\right\}$. We know from Remark $[2.7$ that $\mathscr{C}(R)=\mathcal{G}(R)$. Since $|\operatorname{Max}(R)|=n \geq 3$, it follows from Proposition [2.14 that $r(\mathscr{C}(R))=2$ and $\operatorname{diam}(\mathscr{C}(R))=3$. Therefore, we get that $r(\mathcal{G}(R))=2$ and $\operatorname{diam}(\mathcal{G}(R))=3$.

\section{When is $\mathcal{G}(R)$ a finite complete bipartite graph?}

Let $R$ be a ring such that $|\operatorname{Max}(R)| \geq 2$. The aim of this section is to classify rings $R$ such that $\mathcal{G}(R)$ is a finite complete bipartite graph.

Lemma 3.1. Let $R$ be a ring such that $|\operatorname{Max}(R)| \geq 2$. If $\mathcal{G}(R)$ is bipartite, then $|\operatorname{Max}(R)|=2$.

Proof. It is already noted in the introduction that $\mathscr{C}(R)$ is a spanning subgraph of $\mathcal{G}(R)$. Thus if $\mathcal{G}(R)$ is bipartite, then $\mathscr{C}(R)$ is also a bipartite graph. Hence, we obtain from $(2) \Rightarrow(3)$ of [15, Theorem 4.5] that $|\operatorname{Max}(R)|=2$.

Lemma 3.2. Let $H$ be a spanning subgraph of a graph $G=(V, E)$. Suppose that $H$ is a complete bipartite graph. If $G$ is a bipartite graph, then $H=G$.

Proof. Let $H$ be a complete bipartite graph with vertex partition $V_{1}$ and $V_{2}$. Let $G$ be a bipartite graph with vertex partition $W_{1}$ and $W_{2}$. Note that $V=V_{1} \cup V_{2}=W_{1} \cup W_{2}$ and $V_{1} \cap V_{2}=W_{1} \cap W_{2}=\emptyset$. Let $x \in W_{1}$. Then either $x \in V_{1}$ or $x \in V_{2}$. Without loss of generality, we can assume that $x \in V_{1}$. Let $x^{\prime} \in W_{1}$ be such that $x^{\prime} \neq x$. If $x^{\prime} \in V_{2}$, then $x$ 
and $x^{\prime}$ are adjacent in $H$ and hence, they are adjacent in $G$. This is impossible since $x$ and $x^{\prime}$ are not adjacent in $G$. Therefore, $x^{\prime} \in V_{1}$ and this proves that $W_{1} \subseteq V_{1}$. Let $y \in V_{2}$. Now, $V_{2} \subset V=W_{1} \cup W_{2}$. As $W_{1} \subseteq V_{1}$ and $V_{1} \cap V_{2}=\emptyset$, we obtain that $y \in W_{2}$. This shows that $V_{2} \subseteq W_{2}$. Let $z \in W_{2} \subset V=V_{1} \cup V_{2}$. We claim that $z \in V_{2}$. Suppose that $z \in V_{1}$. Let $y \in V_{2}$. Then $z$ and $y$ are adjacent in $H$ and so, they are adjacent in $G$. As both $z$ and $y$ are in $W_{2}$, they are not adjacent in $G$. This is a contradiction and therefore, $z \in V_{2}$. This shows that $W_{2} \subseteq V_{2}$ and so, $V_{2}=W_{2}$. Hence, we get that $V_{1} \subseteq W_{1}$ and so, $V_{1}=W_{1}$. If $a-b$ is any edge of $G$, then one of $a, b$ must be in $V_{1}$ and the other must be in $V_{2}$. Since $H$ is a complete bipartite graph with vertex partition $V_{1}$ and $V_{2}$, we obtain that $a-b$ is an edge of $H$. This proves that $H=G$.

Corollary 3.3. Let $R$ be a ring such that $|\operatorname{Max}(R)| \geq 2$. If $\mathcal{G}(R)$ is a bipartite graph, then $|\operatorname{Max}(R)|=2, \mathcal{G}(R)$ is a complete bipartite graph and $\mathscr{C}(R)=\mathcal{G}(R)$.

Proof. Assume that $\mathcal{G}(R)$ is a bipartite graph. We know from Lemma Note that $\mathscr{C}(R)$ is a spanning subgraph of $\mathcal{G}(R)$. Thus if $\mathcal{G}(R)$ is a bipartite graph, then $\mathscr{C}(R)$ is also a bipartite graph. In such a case, we get from $(2) \Rightarrow(1)$ of [15, Theorem 4.5] that $\mathscr{C}(R)$ is a complete bipartite graph . Therefore, we obtain from Lemma $\mathbf{3 . 2}$ that $\mathscr{C}(R)=\mathcal{G}(R)$ and so, $\mathcal{G}(R)$ is a complete bipartite graph.

Lemma 3.4. Let $\left(R_{i}, \mathfrak{m}_{i}\right)$ be a quasilocal ring for each $i \in\{1,2\}$ and let $R=R_{1} \times R_{2}$. If $\mathcal{G}(R)$ is a bipartite graph, then $R_{i}$ is a field for each $i \in\{1,2\}$.

Proof. Note that $\left\{\mathfrak{M}_{1}=\mathfrak{m}_{1} \times R_{2}, \mathfrak{M}_{2}=R_{1} \times \mathfrak{m}_{2}\right\}$ is the set of all maximal ideals of $R$. Assume that $\mathcal{G}(R)$ is a bipartite graph. First, we verify that $R_{1}$ is a field. Suppose that $R_{1}$ is not a field. Then $\mathfrak{m}_{1} \neq(0)$. Note that $\mathfrak{m}_{1} \times R_{2}-(0) \times R_{2}-R_{1} \times(0)-\mathfrak{m}_{1} \times R_{2}$ is a cycle of length three in $\mathcal{G}(R)$. This is in contradiction to the assumption that $\mathcal{G}(R)$ is a bipartite graph. Therefore, $R_{1}$ is a field. Similarly, it can be shown that $R_{2}$ is a field. This shows that $R_{i}$ is a field for each $i \in\{1,2\}$.

Let $R$ be a ring. Recall from [ $[\overline{0}]$ that $R$ is said to satisfy descending chain condition on principal powers if for any $a \in R$, the descending sequence of ideals $R a \supseteq R a^{2} \supseteq R a^{3} \supseteq \cdots$ stops after a finite stage.

Let $R$ be a ring such that $|\operatorname{Max}(R)| \geq 2$. In Theorem 3.5, we classify such rings $R$ in order that $\mathcal{G}(R)$ to be a finite bipartite graph. Theorem $[5.5$ of this article is motivated by [15, Proposition 4.7]. 
Alg. Struc. Appl. Vol. 4 No. 1 (2017) 59-78.

Theorem 3.5. Let $R$ be a ring such that $|\operatorname{Max}(R)| \geq 2$. The following statements are equivalent:

(i) $\mathcal{G}(R)$ is a finite bipartite graph.

(ii) $\mathcal{G}(R)$ is a bipartite graph and $R$ satisfies d.c.c. on principal powers on elements $a \in R$ such that $R a \in V(\mathcal{G}(R))$.

(iii) $R \cong F_{1} \times F_{2}$ as rings, where $F_{i}$ is a field for each $i \in\{1,2\}$.

Proof. $(i) \Rightarrow($ ii $)$ As $\mathcal{G}(R)$ is a finite bipartite graph, it follows that $V(\mathcal{G}(R))$ is finite. Let $a \in R$ be such that $R a \in V(\mathcal{G}(R))$. Then $a \in N U(R) \backslash J(R)$. Then $a^{n} \in N U(R) \backslash J(R)$ for all $n \geq 1$ and so, $R a^{n} \in V(\mathcal{G}(R))$ for all $n \geq 1$. Since $V(\mathcal{G}(R))$ is finite, we obtain that there exists $n \geq 1$ such that $R a^{n}=R a^{j}$ for all $j \geq n$.

$($ ii $) \Rightarrow($ iii $)$ Now, by assumption, $\mathcal{G}(R)$ is a bipartite graph. We know from Corollary 3.3 that $|\operatorname{Max}(R)|=2$ and $\mathscr{C}(R)=\mathcal{G}(R)$ is a complete bipartite graph. Let $\left\{\mathfrak{M}_{1}, \mathfrak{M}_{2}\right\}$ denote the set of all maximal ideals of $R$. Let $a \in \mathfrak{M}_{1} \backslash \mathfrak{M}_{2}$. Observe that $R a \in V(\mathcal{G}(R))$. As we are assuming that $R$ satisfies d.c.c. on principal powers on elements $x \in R$ such that $R x \in V(\mathcal{G}(R))$, we get that there exists $n \geq 1$ such that $R a^{n}=R a^{j}$ for all $j \geq n$. This implies that $a^{n}=r a^{2 n}$ for some $r \in R$. Therefore, $e=r a^{n}$ is a nontrivial idempotent element of $R$. Hence, the mapping $f: R \rightarrow R e \times R(1-e)$ defined by $f(x)=(x e, x(1-e))$ is an isomorphism of rings. Let us denote the ring $R e$ by $R_{1}$ and the ring $R(1-e)$ by $R_{2}$. Now, $R \cong R_{1} \times R_{2}$ as rings. Since $|\operatorname{Max}(R)|=2$, it follows that $R_{i}$ is a quasilocal ring for each $i \in\{1,2\}$. As $\mathcal{G}\left(R_{1} \times R_{2}\right)$ is a bipartite graph, we obtain from Lemma $\mathbf{3 . 4}$ that $R_{i}$ is a field for each $i \in\{1,2\}$. Let $i \in\{1,2\}$. With $F_{i}=R_{i}$, we obtain that $F_{i}$ is a field and $R \cong F_{1} \times F_{2}$ as rings.

$($ iii $) \Rightarrow(i)$ Let us denote the ring $F_{1} \times F_{2}$ by $T$, where $F_{i}$ is a field for each $i \in\{1,2\}$. Note that $V(\mathcal{G}(T))=\left\{(0) \times F_{2}, F_{1} \times(0)\right\}$. From $\left((0) \times F_{2}\right)+\left(F_{1} \times(0)\right)=T$, we get that $\mathcal{G}(T)$ is a complete graph on two vertices. Since $R \cong T$ as rings, we obtain that $\mathcal{G}(R)$ is a complete graph on two vertices and hence, it is a finite bipartite graph.

In Example [3.6, we mention an example of a ring $R$ which illustrates that $(i i) \Rightarrow($ iii $)$ of Theorem 3.5 can fail to hold if the hypothesis that $R$ satisfies d.c.c. on principal powers on elements $a \in R$ with $a \in V(\mathcal{G}(R))$ is omitted. The example [3.6] mentioned here is [15, Example 4.10].

Example 3.6. Let $p, q$ be distinct prime numbers. Let $S=\mathbb{Z} \backslash(p \mathbb{Z} \cup q \mathbb{Z})$. Let $R=S^{-1} \mathbb{Z}$. Then $\mathcal{G}(R)$ is a complete bipartite graph and $R$ has no nontrivial idempotent element.

Proof. Note that $R$ is a semilocal principal ideal domain with $\operatorname{Max}(R)=\{p R, q R\}$. We know from Remark 2.7 that $\mathscr{C}(R)=\mathcal{G}(R)$. Since $|\operatorname{Max}(R)|=2$, we know from [1.5, Lemma 4.1] 
that $\mathscr{C}(R)$ is a complete bipartite graph. This shows that $\mathcal{G}(R)$ is a complete bipartite graph. As $R$ is an integral domain, we obtain that 0 and 1 are the only idempotent elements of $R$. Hence, $R \varsubsetneqq R_{1} \times R_{2}$ as rings for any quasilocal rings $R_{1}$ and $R_{2}$.

Let $I$ be an ideal of a ring $R$. As in [1:2], we denote $\{\mathfrak{m} \in \operatorname{Max}(R) \mid \mathfrak{m} \supseteq I\}$ by $M(I)$.

Remark 3.7. Let $R$ be a ring with $|\operatorname{Max}(R)|=2$. Let $\left\{\mathfrak{m}_{1}, \mathfrak{m}_{2}\right\}$ denote the set of all maximal ideals of $R$. We know from [15, Lemma 4.1] that $\mathscr{C}(R)$ is a complete bipartite graph with vertex partition $V_{1}$ and $V_{2}$, where $V_{1}$ is the set of all ideals $I$ of $R$ such that $M(I)=\left\{\mathfrak{m}_{1}\right\}$ and $V_{2}$ is the set of all ideals $J$ of $R$ such that $M(J)=\left\{\mathfrak{m}_{2}\right\}$. It is noted in [15, Corollary 4.2] that $1 \leq \operatorname{diam}(\mathscr{C}(R)) \leq 2$. It is already observed in the introduction that $\mathscr{C}(R)$ is a spanning subgraph of $\mathcal{G}(R)$. Therefore, we obtain that $1 \leq \operatorname{diam}(\mathcal{G}(R)) \leq 2$. In Corollary $\mathbf{B g}$, we classify rings $R$ such that $\operatorname{diam}(\mathcal{G}(R))=1$, that is, we classify rings $R$ such that $\mathcal{G}(R)$ is complete.

Theorem 3.8. Let $\left(R_{i}, \mathfrak{m}_{i}\right)$ be a quasilocal ring for each $i \in\{1,2\}$ and let $R=R_{1} \times R_{2}$. The following statements are equivalent:

(i) $\mathcal{G}(R)$ is complete.

(ii) For each $i \in\{1,2\}, \mathfrak{m}_{i}$ is principal and $\mathfrak{m}_{i}^{2}=(0)$.

Proof. $(i) \Rightarrow($ ii $)$ Assume that $\mathcal{G}(R)$ is complete. First, we verify that $\mathfrak{m}_{1}$ is principal and $\mathfrak{m}_{1}^{2}=(0)$. This is clear if $\mathfrak{m}_{1}=(0)$. Suppose that $\mathfrak{m}_{1} \neq(0)$. Let $a \in \mathfrak{m}_{1} \backslash\{0\}$. We assert that $\mathfrak{m}_{1}=R_{1} a$. If $\mathfrak{m}_{1} \neq R_{1} a$, then the ideals $I_{1}=R_{1} a \times R_{2}$ and $I_{2}=\mathfrak{m}_{1} \times R_{2}$ are distinct members of $V(\mathcal{G}(R))$. Since we are assuming that $\mathcal{G}(R)$ is complete, we get that $I_{1} \cap I_{2}=I_{1} I_{2}$. This implies that $R_{1} a=\mathfrak{m}_{1} a$ and so, $a=x a$ for some $x \in \mathfrak{m}_{1}$. Hence, $a(1-x)=0$. Since $1-x \in U\left(R_{1}\right)$, we get that $a=0$. This is in contradiction to the fact that $a \neq 0$. This proves that $\mathfrak{m}_{1}=R_{1} a$ for any $a \in \mathfrak{m}_{1} \backslash\{0\}$. Let $a \in \mathfrak{m}_{1} \backslash\{0\}$. If $a^{2} \neq 0$, then $R_{1} a=R_{1} a^{2}$. This implies that $a=x a^{2}$ for some $x \in R_{1}$ and so, $a(1-x a)=0$. Since $1-x a \in U\left(R_{1}\right)$, we obtain that $a=0$. This is a contradiction. Therefore, $\mathfrak{m}_{1}$ is principal and $\mathfrak{m}_{1}^{2}=(0)$. Similarly, it can be shown that $\mathfrak{m}_{2}$ is principal and $\mathfrak{m}_{2}^{2}=(0)$.

$($ ii $) \Rightarrow(i)$ Assume that $\mathfrak{m}_{i}$ is principal and $\mathfrak{m}_{i}^{2}=(0)$ for each $i \in\{1,2\}$. Note that $|\operatorname{Max}(R)|=$ 2 and $\left\{\mathfrak{M}_{1}=\mathfrak{m}_{1} \times R_{2}, \mathfrak{M}_{2}=R_{1} \times \mathfrak{m}_{2}\right\}$ is the set of all maximal ideals of $R$. We consider the following cases.

$\operatorname{Case}(1) \mathfrak{m}_{i}=(0)$ for each $i \in\{1,2\}$

In this case, both $R_{1}$ and $R_{2}$ are fields. Note that $V(\mathcal{G}(R))=\left\{(0) \times R_{2}, R_{1} \times(0)\right\}$ and $\left((0) \times R_{2}\right)+\left(R_{1} \times(0)\right)=R$. Hence, $(0) \times R_{2}$ and $R_{1} \times(0)$ are adjacent in $\mathscr{C}(R)$ and so, they are adjacent in $\mathcal{G}(R)$. Therefore, $\mathcal{G}(R)$ is a complete graph on two vertices. 
$\operatorname{Case}(2) \mathfrak{m}_{1} \neq(0)$ but $\mathfrak{m}_{2}=(0)$

Note that $R_{2}$ is a field. As $\mathfrak{m}_{1}$ is a nonzero principal ideal of $R_{1}$ with $\mathfrak{m}_{1}^{2}=(0)$, it follows that $\mathfrak{m}_{1}$ is the only nonzero proper ideal of $R_{1}$. Observe that $V(\mathcal{G}(R))=\left\{(0) \times R_{2}, \mathfrak{m}_{1} \times R_{2}, R_{1} \times(0)\right\}$. Observe that $\mathscr{C}(R)$ is a complete bipartite graph with vertex partition $V_{1}=\left\{\mathfrak{M}_{1}^{2}=(0) \times\right.$ $\left.R_{2}, \mathfrak{M}_{1}=\mathfrak{m}_{1} \times R_{2}\right\}$ and $V_{2}=\left\{\mathfrak{M}_{2}=R_{1} \times(0)\right\}$. As $\mathscr{C}(R)$ is a spanning subgraph of $\mathcal{G}(R)$, it follows that each member of $V_{1}$ is adjacent to each member of $V_{2}$ in $\mathcal{G}(R)$. Observe that $\mathfrak{M}_{1} \cap \mathfrak{M}_{1}^{2}=\mathfrak{M}_{1}^{2}=\mathfrak{M}_{1}^{3}$. Therefore, there is an edge of $\mathcal{G}(R)$ joining $\mathfrak{M}_{1}$ and $\mathfrak{M}_{1}^{2}$. This proves that $\mathcal{G}(R)$ is the cycle $\Gamma$ of length three given by $\Gamma: \mathfrak{M}_{1}-\mathfrak{M}_{2}-\mathfrak{M}_{1}^{2}-\mathfrak{M}_{1}$ and so, $\mathcal{G}(R)$ is a complete graph on three vertices.

$\operatorname{Case}(3) \mathfrak{m}_{1}=(0)$ but $\mathfrak{m}_{2} \neq(0)$

Since $\mathfrak{m}_{2}$ is a nonzero principal ideal of $R_{2}$ with $\mathfrak{m}_{2}^{2}=(0)$, it follows as in Case $(2)$ that $\mathcal{G}(R)$ is a complete graph on three vertices.

$\operatorname{Case}(4) \mathfrak{m}_{i} \neq(0)$ for each $i \in\{1,2\}$

Let $i \in\{1,2\}$. Since $\mathfrak{m}_{i}$ is a nonzero principal ideal of $R_{i}$ with $\mathfrak{m}_{i}^{2}=(0)$, it follows that $\mathfrak{m}_{i}$ is the only nonzero proper ideal of $R_{i}$. Note that $V(\mathcal{G}(R))=\left\{\mathfrak{M}_{1}=\mathfrak{m}_{1} \times R_{2}, \mathfrak{M}_{1}^{2}=\right.$ $\left.(0) \times R_{2}, \mathfrak{M}_{2}=R_{1} \times \mathfrak{m}_{2}, \mathfrak{M}_{2}^{2}=R_{1} \times(0)\right\}$. Observe that $\mathscr{C}(R)$ is a complete bipartite graph with vertex partition $V_{1}=\left\{\mathfrak{M}_{1}, \mathfrak{M}_{1}^{2}\right\}$ and $V_{2}=\left\{\mathfrak{M}_{2}, \mathfrak{M}_{2}^{2}\right\}$. Since $\mathscr{C}(R)$ is a spanning subgraph of $\mathcal{G}(R)$, it follows that each member of $V_{1}$ is adjacent to each member of $V_{2}$ in $\mathcal{G}(R)$. Moreover, it follows as in Case $(2)$ that $\mathfrak{M}_{i}-\mathfrak{M}_{i}^{2}$ is an edge of $\mathcal{G}(R)$ for each $i \in\{1,2\}$. Therefore, we get that $\mathcal{G}(R)$ is a complete graph on four vertices.

This proves that $\mathcal{G}(R)$ is complete.

Corollary 3.9. Let $R$ be a ring such that $|\operatorname{Max}(R)|=2$. The following statements are equivalent:

(i) $\mathcal{G}(R)$ is complete.

(ii) $R$ is isomorphic to one of the following rings:

(a) $F_{1} \times F_{2}$, where $F_{i}$ is a field for each $i \in\{1,2\}$.

(b) $F_{1} \times R_{2}$, where $F_{1}$ is a field and $\left(R_{2}, \mathfrak{m}_{2}\right)$ is a SPIR with $\mathfrak{m}_{2} \neq(0)$ but $\mathfrak{m}_{2}^{2}=(0)$.

(c) $R_{1} \times R_{2}$, where $\left(R_{i}, \mathfrak{m}_{i}\right)$ is a SPIR with $\mathfrak{m}_{i} \neq(0)$ but $\mathfrak{m}_{i}^{2}=(0)$ for each $i \in\{1,2\}$.

Proof. $(i) \Rightarrow($ ii $)$ Let $\left\{\mathfrak{M}_{1}, \mathfrak{M}_{2}\right\}$ denote the set of all maximal ideals of $R$. We assert that $R$ admits at least one nontrivial idempotent. Let $a \in \mathfrak{M}_{1} \backslash \mathfrak{M}_{2}$. Then for all $n \geq 2, a^{n} \in \mathfrak{M}_{1} \backslash \mathfrak{M}_{2}$. Hence, $R a^{n} \in V(\mathcal{G}(R))$ for all $n \geq 1$. If $R a=R a^{2}$, then $a=r a^{2}$ for some $r \in R$. In such a case, $r a$ is a nontrivial idempotent element of $R$. Suppose that $R a \neq R a^{2}$. Since $\mathcal{G}(R)$ is complete, the vertices $R a$ and $R a^{2}$ are adjacent in $\mathcal{G}(R)$. Hence, $R a \cap R a^{2}=R a^{3}$ and so, $R a^{2}=R a^{3}$. Therefore, $R a^{2}=R a^{4}$. This implies that $a^{2}=s a^{4}$ for some $s \in R$ and so, $s a^{2}$ is a nontrivial idempotent element of $R$. This shows that there exists a nontrivial idempotent 
element $e$ of $R$. Note that the mapping $f: R \rightarrow R e \times R(1-e)$ defined by $f(r)=(r e, r(1-e))$ is an isomorphism of rings. Let us denote the ring $R e$ by $R_{1}$ and the ring $R(1-e)$ by $R_{2}$. Let us denote the ring $R_{1} \times R_{2}$ by $T$. Since $R \cong T$ as rings, we obtain that $\mathcal{G}(T)$ is complete. As $|\operatorname{Max}(T)|=2$, it follows that $R_{i}$ admits a unique maximal ideal for each $i \in\{1,2\}$. Let $\mathfrak{m}_{i}$ denote the unique maximal ideal of $R_{i}$ for each $i \in\{1,2\}$. Now, we know from $(i) \Rightarrow(i i)$ of Theorem 3.8 that $\mathfrak{m}_{i}$ is principal and $\mathfrak{m}_{i}^{2}=(0)$ for each $i \in\{1,2\}$. If $\mathfrak{m}_{i}=(0)$ for each $i \in\{1,2\}$, then $R_{i}$ is a field for each $i \in\{1,2\}$. With $F_{i}=R_{i}$ for each $i \in\{1,2\}$, we get that $R$ is isomorphic to the ring mentioned in $(i i)(a)$. Suppose that exactly one between $\mathfrak{m}_{1}$ and $\mathfrak{m}_{2}$ is the zero ideal. Without loss of generality, we can assume that $\mathfrak{m}_{1}=(0)$. Then $R_{1}$ is a field and $\left(R_{2}, \mathfrak{m}_{2}\right)$ is a SPIR with $\mathfrak{m}_{2} \neq(0)$ but $\mathfrak{m}_{2}^{2}=(0)$. In this case, with $F_{1}=R_{1}$, we obtain that $R$ is isomorphic to the ring mentioned in $(i i)(b)$. If $\mathfrak{m}_{i} \neq(0)$ for each $i \in\{1,2\}$, then $\left(R_{i}, \mathfrak{m}_{i}\right)$ is a SPIR with $\mathfrak{m}_{i} \neq(0)$ but $\mathfrak{m}_{i}^{2}=(0)$ for each $i \in\{1,2\}$. and $R$ is isomorphic to the ring mentioned in $(i i)(c)$.

$($ ii $) \Rightarrow(i)$ Assume that $R$ is isomorphic to one of the rings mentioned in $(i i)(a),(b)$ or $(c)$. Let $F_{1}, F_{2}$ be fields. Let us denote $F_{1} \times F_{2}$ by $T_{1}$. We know from the proof of $(i i) \Rightarrow(i)$ Case(1) of Theorem 3.8 that $\mathcal{G}\left(T_{1}\right)$ is a complete graph on two vertices. Let $F_{1}$ be a field and $\left(R_{2}, \mathfrak{m}_{2}\right)$ be a SPIR with $\mathfrak{m}_{2} \neq(0)$ but $\mathfrak{m}_{2}^{2}=(0)$. Let us denote the ring $F_{1} \times R_{2}$ by $T_{2}$. We know from the proof of $(i i) \Rightarrow(i)$ Case(3) of Theorem 3.8 that $\mathcal{G}\left(T_{2}\right)$ is a complete graph on three vertices. Suppose that $\left(R_{i}, \mathfrak{m}_{i}\right)$ be a SPIR with $\mathfrak{m}_{i} \neq(0)$ but $\mathfrak{m}_{i}^{2}=(0)$ for each $i \in\{1,2\}$. Let us denote the ring $R_{1} \times R_{2}$ by $T_{3}$. We know from the proof of $(i i) \Rightarrow(i)$ Case(4) of Theorem 3.8 that $\mathcal{G}\left(T_{3}\right)$ is a complete graph on four vertices. This proves that $\mathcal{G}(R)$ is complete.

\section{On the clique number and the chromatic number of $\mathcal{G}(R)$}

Let $R$ be a ring with $|\operatorname{Max}(R)| \geq 2$. The aim of this section is to discuss some results regarding $\omega(\mathcal{G}(R))$ and $\chi(\mathcal{G}(R))$.

Remark 4.1. Let $R$ be a ring with $|\operatorname{Max}(R)| \geq 2$. Suppose that $\omega(\mathcal{G}(R))<\infty$. It is already noted in the introduction that $\mathscr{C}(R)$ is a spanning subgraph of $\mathcal{G}(R)$. Hence, $\omega(\mathscr{C}(R))$ is also finite. If $\omega(\mathscr{C}(R))=n$, then we know from [15, Theorem 3.1] that $|\operatorname{Max}(R)|=n=\chi(\mathscr{C}(R))$. Thus if $\omega(\mathcal{G}(R))<\infty$, then $R$ is semiquasilocal. In Example 4.2, we provide an example of a semiquasilocal ring $R$ such that $\mathcal{G}(R)$ admits an infinite clique.

Example 4.2. Let $V$ be an infinite dimensional vector space over a field $K$. Let $T=K \oplus V$ be the ring obtained on using Nagata's principle of idealization. Let $R=T \times T$. Then $|\operatorname{Max}(R)|=2$ and $\mathcal{G}(R)$ admits an infinite clique. 
Proof. Note that $T$ is quasilocal with $\mathfrak{m}=(0) \oplus V$ as its unique maximal ideal and $\mathfrak{m}^{2}=$ $(0) \oplus(0)$. Hence, $R=T \times T$ has $\left\{\mathfrak{M}_{1}=\mathfrak{m} \times T, \mathfrak{M}_{2}=T \times \mathfrak{m}\right\}$ as its set of all maximal ideals. This shows that $|\operatorname{Max}(R)|=2$. Since $V$ is an infinite dimensional vector space over $K$, it is possible to find $v_{i} \in V$ for each $i \in \mathbb{N}$ such that $\left\{v_{i} \mid i \in \mathbb{N}\right\}$ is linearly independent over $K$. For each $i \in \mathbb{N}$, let us denote the ideal $(0) \oplus K v_{i}$ of $T$ by $I_{i}$. Let $i, j \in \mathbb{N}, i \neq j$. Since $v_{i}, v_{j}$ are linearly independent over $K$, it follows that $K v_{i} \cap K v_{j}=(0)$ and so, $I_{i} \cap I_{j}=(0) \oplus(0)$. Hence, $I_{i} I_{j}=I_{i} \cap I_{j}=(0) \oplus(0)$. For each $i \in \mathbb{N}$, let us denote the ideal $I_{i} \times T$ of $R$ by $A_{i}$. It is clear that $A_{i}$ is a proper ideal of $R$ and $A_{i} \nsubseteq J(R)$ for each $i \in \mathbb{N}$. Hence, $A_{i} \in V(\mathcal{G}(R))$ for each $i \in \mathbb{N}$. Let $i, j$ be distinct elements of $\mathbb{N}$. From $I_{i} I_{j}=I_{i} \cap I_{j}$, it follows that $A_{i} A_{j}=I_{i} I_{j} \times T=\left(I_{i} \cap I_{j}\right) \times T=A_{i} \cap A_{j}$. Hence, the subgraph of $\mathcal{G}(R)$ induced on $\left\{A_{i} \mid i \in \mathbb{N}\right\}$ is an infinite clique.

In Proposition 4.3 we classify rings $R$ with $|\operatorname{Max}(R)| \geq 2$ and $J(R)=(0)$ such that $\omega(\mathcal{G}(R))<\infty$.

Proposition 4.3. Let $R$ be a ring such that $|\operatorname{Max}(R)| \geq 2$ and suppose that $J(R)=(0)$. The following statements are equivalent:

(i) $\omega(\mathcal{G}(R))<\infty$.

(ii) $\mathcal{G}(R)$ does not contain any infinite clique.

(iii) There exist $n \in \mathbb{N}$ with $n \geq 2$ and fields $F_{1}, F_{2} \ldots, F_{n}$ such that $R \cong F_{1} \times F_{2} \times \cdots \times F_{n}$ as rings.

Moreover, if any one of the statements (i), (ii) or (iii) holds (and hence, all the three hold), then $\omega(\mathcal{G}(R))=\chi(\mathcal{G}(R))=2^{n}-2$.

Proof. $(i) \Rightarrow($ ii $)$ This is clear.

$($ ii $) \Rightarrow($ iii $)$ We claim that $\operatorname{Max}(R)$ is finite. Suppose that $\operatorname{Max}(R)$ is infinite. If $\mathfrak{m}$ is any element of $\operatorname{Max}(R)$, then $\mathfrak{m} \in V(\mathcal{G}(R))$. Now, for any distinct $\mathfrak{m}, \mathfrak{m}^{\prime} \in \operatorname{Max}(R), \mathfrak{m}+\mathfrak{m}^{\prime}=R$. Hence, $\mathfrak{m}$ and $\mathfrak{m}^{\prime}$ are adjacent in $\mathscr{C}(R)$ and so, they are adjacent in $\mathcal{G}(R)$. Therefore, the subgraph of $\mathcal{G}(R)$ induced on $\operatorname{Max}(R)$ is an infinite clique. This is in contradiction to the assumption that $\mathcal{G}(R)$ does not contain any infinite clique. Hence, $\operatorname{Max}(R)$ is finite. Let $|\operatorname{Max}(R)|=n$. It is clear that $n \geq 2$. Let $\left\{\mathfrak{m}_{1}, \mathfrak{m}_{2}, \ldots, \mathfrak{m}_{n}\right\}$ denote the set of all maximal ideals of $R$. By hypothesis, $J(R)=(0)$. Hence, $\cap_{i=1}^{n} \mathfrak{m}_{i}=(0)$. Now, it follows from the Chinese remainder theorem [2, Proposition 1.10(ii) and (iii)] that the mapping $f: R \rightarrow$ $\frac{R}{\mathfrak{m}_{1}} \times \frac{R}{\mathfrak{m}_{2}} \times \cdots \times \frac{R}{\mathfrak{m}_{n}}$ given by $f(r)=\left(r+\mathfrak{m}_{1}, r+\mathfrak{m}_{2}, \ldots, r+\mathfrak{m}_{n}\right)$ is an isomorphism of rings. For each $i \in\{1,2, \ldots, n\}$, let us denote the field $\frac{R}{\mathfrak{m}_{i}}$ by $F_{i}$. It is clear that $R \cong F_{1} \times F_{2} \times \cdots \times F_{n}$ as rings. 
$($ iii $) \Rightarrow\left(\right.$ i) Let us denote the ring $F_{1} \times F_{2} \times \cdots \times F_{n}$ by $T$. Note that $T$ is a von Neumann regular ring. Hence, we obtain from $(i i) \Rightarrow(i)$ of Theorem 2.4 that $\mathcal{G}(T)$ is complete. Since $V(\mathcal{G}(T))$ is the set of all nonzero proper ideals of $T$, it follows that $\mathcal{G}(T)$ is a complete graph on $2^{n}-2$ vertices. From $R \cong T$ as rings, we get that $\mathcal{G}(R)$ is a complete graph on $2^{n}-2$ vertices and so, $\omega(\mathcal{G}(R))=\chi(\mathcal{G}(R))=2^{n}-2$.

The moreover part of this proposition is already verified in $(i i i) \Rightarrow(i)$ of this proposition.

In Example 4.4, we provide an example of a ring $R$ such that $|\operatorname{Max}(R)|=2$ with $\omega(\mathcal{G}(R))=$ $5<\chi(\mathcal{G}(R))=6$.

Example 4.4. Let $T=\mathbb{Z}_{4}[X, Y, Z]$ be the polynomial ring in three variables $X, Y, Z$ over $\mathbb{Z}_{4}$. Let $I$ be the ideal of $T$ generated by $\left\{X^{2}-2, Y^{2}-2, Z^{2}, X Y, Y Z-2, X Z, 2 X, 2 Y, 2 Z\right\}$. Let $S=\frac{T}{I}$ and let $R=S \times F$, where $F$ is a field. Then $|\operatorname{Max}(R)|=2$ and $\omega(\mathcal{G}(R))=5<$ $\chi(\mathcal{G}(R))=6$.

Proof. The ring $S$ mentioned in Example 4.4 is an interesting and inspiring example due to D.D. Anderson and M. Nasser [G] which answered a conjecture of I. Beck [4] in the negative. It was already noted in [I] that $S$ is a finite local ring with $\mathfrak{m}=\frac{T X+T Y+T Z}{I}$ as its unique maximal ideal, $\mathfrak{m}^{2}=S(2+I)=\{0+I, 2+I\}$, and $\mathfrak{m}^{3}=(0+I)$. Since $R=S \times F$, it is clear that $|\operatorname{Max}(R)|=2$ and $\left\{\mathfrak{M}_{1}=\mathfrak{m} \times F, \mathfrak{M}_{2}=S \times(0)\right\}$ is the set of all maximal ideals of $R$. It is convenient to denote $X+I$ by $x, Y+I$ by $y$, and $Z+I$ by $z$. It was already observed in the proof of [6, Proposition 2.1] that the set of all nonzero proper ideals of $S$ equals $\{S(2+I), S x, S y, S z, S(x+y), S(y+z), S(x+z), S(x+y+z), S x+S y, S y+$ $S z, S x+S z, S x+S(y+z), S y+S(z+x), S z+S(x+y), S x+S y+S z\}$. Observe that $\mathfrak{m}^{2}=S(2+I)$ is the unique minimal ideal of $S$. Note that $J(R)=\mathfrak{m} \times(0)$ and $V(\mathcal{G}(R))$ equals $\{\mathfrak{m} \times F,(0+I) \times F, S \times(0), S x \times F, S y \times F, S z \times F, S(x+y) \times F, S(y+z) \times F, S(x+z) \times$ $F, S(x+y+z) \times F,(S x+S y) \times F,(S y+S z) \times F,(S x+S z) \times F,(S x+S(y+z)) \times F,(S y+$ $S(x+z)) \times F,(S z+S(x+y)) \times F, S(2+I) \times F\}$.

We next proceed to verify that $\omega(\mathcal{G}(R)) \leq 5$. It is convenient to denote $\{S x \times F, S y \times F, S z \times$ $F\}$ by $W_{1},\{S(x+y) \times F, S(y+z) \times F, S(x+z) \times F,(S x+S y) \times F,(S y+S z) \times F,(S x+S z) \times F\}$ by $W_{2}$, and $\{(S x+S(y+z)) \times F,(S y+S(x+z)) \times F,(S z+S(x+y)) \times F\}$ by $W_{3}$. Let $W \subseteq V(\mathcal{G}(R))$ be such that the subgraph of $\mathcal{G}(R)$ induced on $W$ is a clique.

Suppose that $\mathfrak{m}^{2} \times F=S(2+I) \times F \in W$. As $\mathfrak{m}^{2}$ is the unique minimal ideal of $S$, it follows that for any nonzero proper ideal $B$ of $S, \mathfrak{m}^{2} \cap B=\mathfrak{m}^{2}$ but $\mathfrak{m}^{2} B=(0+I)$. Hence, $S(2+I) \times F$ is not adjacent in $\mathcal{G}(R)$ to any vertex of the form $B \times F$, where $B$ is a nonzero proper ideal of $S$. Hence, the only possible vertices that can belong to $W$ are $\mathfrak{m}^{2} \times F,(0+I) \times F$, and $S \times(0)$. Thus if $S(2+I) \times F \in W$, then $|W| \leq 3$. 
Hereafter, we assume that $\mathfrak{m}^{2} \times F \notin W$.

Suppose that $\mathfrak{m} \times F \in W$. Let $B_{1}, B_{2}$ be distinct nonzero proper ideals of $S$ such that $B_{1} \cap B_{2} \nsubseteq \mathfrak{m}^{2}$. Note that $B_{i} \nsubseteq \mathfrak{m}^{2}$ for each $i \in\{1,2\}$. Then $\left(B_{1} \times F\right) \cap\left(B_{2} \times F\right)=$ $\left(B_{1} \cap B_{2}\right) \times F \nsubseteq \mathfrak{m}^{2} \times F$, whereas $\left(B_{1} \times F\right)\left(B_{2} \times F\right)=B_{1} B_{2} \times F \subseteq \mathfrak{m}^{2} \times F$. Hence, $B_{1} \times F$ and $B_{2} \times F$ are not adjacent in $\mathcal{G}(R)$. Therefore, at most one between $B_{1} \times F$ and $B_{2} \times F$ can be in $W$. In particular, if $C_{1}$ and $C_{2}$ are nonzero proper ideals of $S$ with $C_{i} \not \mathfrak{m}^{2}$ for each $i \in\{1,2\}$ and $C_{1} \subset C_{2}$, then at most one between $C_{1} \times F$ and $C_{2} \times F$ can be in $W$. Thus if $\mathfrak{m} \times F \in W$, then the possible vertices that can be in $W$ are $\mathfrak{m} \times F, S \times(0)$, and $(0) \times F$. Therefore, $|W| \leq 3$.

Hereafter, we assume that $\mathfrak{m} \times F \notin W$.

Since $S$ is a local ring, it follows that if $m_{1}, m_{2} \in \mathfrak{m} \backslash \mathfrak{m}^{2}$ are such that $S m_{1} \neq S m_{2}$, then $S m_{1} \cap S m_{2} \subseteq \mathfrak{m}^{2}$. Hence, if $m_{1} m_{2} \neq 0+I$, then $S m_{1} m_{2}=S m_{1} \cap S m_{2}$. Therefore, $S m_{1} \times F$ and $S m_{2} \times F$ are adjacent in $\mathcal{G}(R)$. If $m_{1} m_{2}=(0)+I$, then as $\mathfrak{m}^{2} \times F \subseteq\left(S m_{1} \times F\right) \cap$ $\left(S m_{2} \times F\right)$, it follows that $S m_{1} \times F$ and $S m_{2} \times F$ are not adjacent in $\mathcal{G}(R)$. Since $S y \neq S z$ and $y z=2+I \neq 0+I$, we get that $S y \times F$ and $S z \times F$ are adjacent in $\mathcal{G}(R)$. Observe that $\mathfrak{m}^{2} \times F \subseteq(S x \times F) \cap(S y \times F) \cap(S z \times F)$, whereas $S x y \times F=(0+I) \times F=S x z \times F$. Hence, we get that $(S x \times F) \cap(S y \times F) \neq S x y \times F$ and $(S x \times F) \cap(S z \times F) \neq S x z \times F$ and so, in $\mathcal{G}(R), S x \times F$ is not adjacent to any of the members from $\{S y \times F, S z \times F\}$. Thus if either $S y \times F \in W$ or $S z \times F \in W$, then $S x \times F$ cannot be in $W$.

As $(x+y)(y+z)=0+I$, at most one between $S(x+y) \times F$ and $S(y+z) \times F$ can be in $W$. Hence, $|W \cap\{S(x+y) \times F, S(y+z) \times F, S(x+z) \times F\}| \leq 2$. It is clear that at most one among $(S x+S y) \times F,(S y+S z) \times F$, and $(S x+S z) \times F$ can belong to $W$. Note that $(x+y)(x+z)=0+I$ and so, $S(x+y) \times F$ and $S(x+z) \times F$ are not adjacent in $\mathcal{G}(R)$. It follows from $(y+z)(x+z)=2+I \neq 0+I$ that $S(y+z) \times F$ and $S(x+z) \times F$ are adjacent in $\mathcal{G}(R)$. Suppose that both $S(y+z) \times F$ and $S(x+z) \times F$ are in $W$. Then no member from $\{(S x+S y) \times F,(S y+S z) \times F,(S x+S z) \times F\}$ can belong to $W$. This shows that $\left|W \cap W_{2}\right| \leq 2$.

Note that $x+y+z$ is in each member of $W_{3}$. Hence, at most one element of $W_{3}$ can belong to $W$. Moreover, if $S(x+y+z) \times F \in W$, then $W \cap W_{3}=\emptyset$.

Suppose that both $S y \times F$ and $S z \times F$ are in $W$. Note that $y(y+z)=y^{2}+y z=$ $(2+I)+(2+I)=0+I$. Observe that $0+I=x y=x z=y(y+z)=y(x+y+z)=z(x+z)$. Also, as $S y \times F \subset(S x+S y) \times F, S y \times F \subset(S y+S z) \times F$, and $S z \times F \subset(S x+S z) \times F$, it follows that the only possible member of $W_{2}$ that can belong to $W$ is $S(x+y) \times F$. Moreover, it is clear that $S x \times F$ and $S(x+y+z) \times F$ cannot be in $W$ and furthermore, $W \cap W_{3}=\emptyset$. From the above given arguments, it is clear that the only possible members of $V(\mathcal{G}(R))$ that can be in $W$ are $S y \times F, S z \times F, S(x+y) \times F,(0) \times F$, and $S \times(0)$. Hence, $|W| \leq 5$.

Hereafter, we assume that at most one between $S y \times F$ and $S z \times F$ is in $W$. 
Suppose that $S y \times F \in W$ but $S z \times F \notin W$.

It follows as in the previous paragraph that none of the members from $\{S x \times F, S(y+z) \times$ $F, S(x+y+z) \times F,(S x+S y) \times F,(S y+S z) \times F,(S x+S(y+z)) \times F,(S y+S(x+z)) \times F\}$ can be in $W$. Note that $y(x+y)=y(x+z)=2+I \neq 0+I$ and $(x+y)(x+z)=0+I$. It is clear that at most two members from $\{S(x+y) \times F, S(x+z) \times F,(S x+S z) \times F,(S z+S(x+y)) \times F\}$ can be in $W$. From the above discussion, it follows that $W \subseteq\{S y \times F\} \cup(W \cap\{S(x+y) \times$ $F, S(x+z) \times F,(S x+S z) \times F,(S z+S(x+y)) \times F\} \cup\{(0) \times F, S \times(0)\}$. Therefore, we obtain that $|W| \leq 5$.

Suppose that $S z \times F \in W$ but $S y \times F \notin W$.

It follows from the reasons mentioned earlier in the verification of this example that none of the members from $\{S x \times F, S(x+z) \times F,(S y+S z) \times F,(S x+S z) \times F,(S z+S(x+y)) \times F\}$ can be in $W$. Observe that $z(x+y)=z(y+z)=2+I \neq 0+I$. Hence, we obtain that the possible members from $W_{2}$ that can belong to $W$ are $S(x+y) \times F,(S x+S y) \times F, S(y+z) \times F$ and so, we obtain that $\left|W \cap W_{2}\right| \leq 1$. Note that $z(x+y+z)=2+I \neq 0+I$. Hence, $S z \times F$ and $S(x+y+z) \times F$ are adjacent in $\mathcal{G}(R)$. If $S(x+y+z) \times F \in W$, then it is already observed that $W \cap W_{3}=\emptyset$. Thus in this case, $W \subseteq\{S z \times F, S(x+y+z) \times F\} \cup\left(W \cap W_{2}\right) \cup\{(0) \times F, S \times(0)\}$ and so, $|W| \leq 5$. Suppose that $S(x+y+z) \times F \notin W$. It is already noted that $\left|W \cap W_{3}\right| \leq 1$. In this case, $W \subseteq\{S z \times F\} \cup\left(W \cap W_{2}\right) \cup\left(W \cap W_{3}\right) \cup\{(0) \times F, S \times(0)\}$. Therefore, we obtain that $|W| \leq 5$.

Hereafter, we assume that both $S y \times F$ and $S z \times F$ are not in $W$.

Suppose that $S x \times F \in W$. Then none of the members from $\{S(y+z) \times F,(S x+S y) \times F,(S x+$ $S z) \times F,(S y+S z) \times F,(S x+S(y+z)) \times F\}$ can belong to $W$. It is easy to verify that the possible members from $W_{2}$ that can be in $W$ are $S(x+y) \times F$ and $S(x+z) \times F$. Since $S(x+y) \times F$ and $S(x+z) \times F$ are not adjacent in $\mathcal{G}(R)$, we obtain that $\left|W \cap W_{2}\right| \leq 1$. From $x(x+y+z) \neq 0+I$, it follows that $S x \times I$ and $S(x+y+z) \times F$ are adjacent in $\mathcal{G}(R)$. If $S(x+y+z) \times F \in W$, then $W \cap W_{3}=\emptyset$. Therefore, in this case, $W \subseteq\{S x \times F, S(x+y+z) \times F\} \cup\left(W \cap W_{2}\right) \cup\{(0) \times F, S \times(0)\}$ and so, $|W| \leq 5$. Suppose that $S(x+y+z) \times F \notin W$. It is already noted that $\left|W \cap W_{3}\right| \leq 1$. Hence, in this case, we get that $W \subseteq\{S x \times F\} \cup\left(W \cap W_{2}\right) \cup\left(W \cap W_{3}\right) \cup\{(0) \times F, S \times(0)\}$ and so, $|W| \leq 5$.

Hereafter, we assume that $S x \times F \notin W$.

Note that $\left|W \cap W_{2}\right| \leq 2$ and $\left|W \cap W_{3}\right| \leq 1$. If $S(x+y+z) \times F \in W$, then $W \cap W_{3}=\emptyset$ and in this case, $W \subseteq\{S(x+y+z) \times F\} \cup\left(W \cap W_{2}\right) \cup\{(0) \times F, S \times(0)\}$ and so, $|W| \leq 5$. Suppose that $S(x+y+z) \times F \notin W$. Then as $W \subseteq\left(W \cap W_{2}\right) \cup\left(W \cap W_{3}\right) \cup\{(0) \times F, S \times(0)\}$, it follows that $|W| \leq 5$.

This proves that if $W$ is any subset of $V(\mathcal{G}(R))$ such that the subgraph of $\mathcal{G}(R)$ induced on $W$ is a clique, then $|W| \leq 5$. 
Observe that the subgraph of $\mathcal{G}(R)$ induced on $\{S x \times F, S(x+y) \times F, S(x+y+z) \times$ $F,(0) \times F, S \times(0)\}$ is a clique on five vertices and so, $\omega(\mathcal{G}(R)) \geq 5$. Therefore, we obtain that $\omega(\mathcal{G}(R))=5$.

Now, $\chi(\mathcal{G}(R)) \geq \omega(\mathcal{G}(R))=5$. We claim that $\chi(\mathcal{G}(R))>5$. Suppose that $\chi(\mathcal{G}(R))=5$. Then the vertices of $\mathcal{G}(R)$ can be properly colored using a set of five distinct colors. Let $\left\{c_{1}, c_{2}, c_{3}, c_{4}, c_{5}\right\}$ be a set of five distinct colors that are used for a proper coloring of the vertices of $\mathcal{G}(R)$. Let $i \in\{1,2,3,4,5\}$ and let $V_{i}=\left\{I \in V(\mathcal{G}(R)) \mid I\right.$ receives color $\left.c_{i}\right\}$. Note that $V_{i} \neq \emptyset$ for each $i \in\{1,2,3,4,5\}, V_{i} \cap V_{j}=\emptyset$ for all distinct $i, j \in\{1,2,3,4,5\}$, and $V(\mathcal{G}(R))=\cup_{i=1}^{5} V_{i}$. Since the subgraph of $\mathcal{G}(R)$ induced on $W=\{(0) \times F, S \times(0), S x \times F, S(x+y) \times F, S(x+y+z) \times$ $F\}$ is a clique, no two members from $W$ can be in the same $V_{i}$ for any $i \in\{1,2,3,4,5\}$. Without loss of generality, we can assume that $(0) \times F \in V_{1}, S \times(0) \in V_{2}, S x \times F \in V_{3}, S(x+y) \times F \in V_{4}$, and $S(x+y+z) \times F \in V_{5}$. Since $(0) \times F$ (respectively, $\left.S \times(0)\right)$ is adjacent in $\mathcal{G}(R)$ to all of its other vertices, we obtain that $V_{1}=\{(0) \times F\}$ and $V_{2}=\{S \times(0)\}$. Since $S z \times F$ is adjacent to both $S(x+y) \times F$ and $S(x+y+z) \times F$ but it is not adjacent to $S x \times F$ in $\mathcal{G}(R)$, we get that $S z \times F$ must be in $V_{3}$. Note that $S(x+z) \times F$ is adjacent to $S x \times F$ and is not adjacent to any of the members from $\{S(x+y) \times F, S(x+y+z) \times F\}$ in $\mathcal{G}(R)$. Therefore, $S(x+z) \times F \in V_{4} \cup V_{5}$. Suppose that $S(x+z) \times F \in V_{4}$. As $S(y+z) \times F$ is adjacent to each one of the members from $\{S z \times F, S(x+z) \times F, S(x+y+z) \times F\}$ in $\mathcal{G}(R), S(y+z) \times F \notin \cup_{i=1}^{5} V_{i}$. This is a contradiction. Suppose that $S(x+z) \times F \in V_{5}$. Since $S y \times F$ is adjacent to each one of the members from $\{S z \times F, S(x+y) \times F, S(x+z) \times F\}$ in $\mathcal{G}(R)$, we get that $S y \times F \notin \cup_{i=1}^{5} V_{i}$. This is a contradiction. Therefore, $\chi(\mathcal{G}(R)) \geq 6$.

We next verify that $\chi(\mathcal{G}(R)) \leq 6$. Let $\left\{c_{1}, c_{2}, c_{3}, c_{4}, c_{5}, c_{6}\right\}$ be a set consisting of six distinct colors. We now show that the vertices of $\mathcal{G}(R)$ can be properly colored using $\left\{c_{1}, c_{2}, c_{3}, c_{4}, c_{5}, c_{6}\right\}$. Let us assign the color $c_{1}$ to $(0) \times F$, the color $c_{2}$ to $S \times(0)$, the color $c_{3}$ to $S x \times F$, the color $c_{4}$ to $S(x+y) \times F$, the color $c_{5}$ to $S(x+y+z) \times F$, the color $c_{3}$ to each one of the members from $\left\{S z \times F,(S x+S z) \times F, \mathfrak{m} \times F, \mathfrak{m}^{2} \times F\right\}$, the color $c_{4}$ to each one of the members from $\{S(x+z) \times F,(S z+S(x+y)) \times F\}$, the color $c_{5}$ to each one of the members from $\{S y \times F,(S y+S(x+z)) \times F\}$, and the color $c_{6}$ to each one of the members from $\{(S x+S y) \times F, S(y+z) \times F,(S y+S z) \times F,(S x+S(y+z)) \times F\}$. Note that the above assignment of six colors to the vertices of $\mathcal{G}(R)$ is proper and so, $\chi(\mathcal{G}(R)) \leq 6$. Therefore, we obtain that $\chi(\mathcal{G}(R))=6$.

\section{AcKnowledgments}

We are very much thankful to the referee for many useful suggestions. 
Alg. Struc. Appl. Vol. 4 No. 1 (2017) 59-78.

\section{REFERENCES}

[1] D.D. Anderson and M. Naseer, Beck's coloring of a commutative ring, J. Algebra. 159 (2) (1993), 500-514.

[2] M.F. Atiyah and I.G. Macdonald, Introduction to Commutative Algebra, Addison-Wesley, Reading, Massachusetts, (1969).

[3] R. Balakrishnan and K. Ranganathan, A Textbook of Graph Theory, Universitext, Springer, (2000).

[4] I. Beck, Coloring of commutative rings, J. Algebra. 116 (1988), 208-226.

[5] M. Behboodi and Z. Rakeei, The annihilating-ideal graph of commutative rings I , J. Algebra Appl. 10 (4) (2011), 727-739.

[6] M. Behboodi and Z. Rakeei, The annihilating-ideal graph of commutative rings II , J. Algebra Appl. 10 (4) (2011), 741-753.

[7] R. Gilmer, Multiplicative Ideal Theory, Marcel-Dekker, New York, (1972).

[8] C. Gottlieb, Strongly prime ideals and strongly zero-dimensional rings, J. Algebra Appl. 16 (10) (2017) Article ID. 1750191 (9 pages).

[9] M.I. Jinnah and S.C. Mathew, When is the comaximal graph split?, Comm. Algebra. 40 (7) (2012), 24002404.

[10] H.R. Maimani, M. Salimi, A. Sattari, and S. Yassemi, Comaximal graph of commutative rings, J. Algebra. 319 (2008), 1801-1808.

[11] S.M. Moconja and Z.Z. Petrovic, On the structure of comaximal graphs of commutative rings with identity, Bull. Aust. Math. Soc. 83 (2011), 11-21.

[12] K. Samei, On the comaximal graph of a commutative ring, Canad. Math. Bull. 57 (2) (2014), 413-423.

[13] P.K. Sharma and S.M. Bhatwadekar, A note on graphical representation of rings, J. Algebra. 176 (1995), 124-127.

[14] H.J. Wang, Graphs associated to co-maximal ideals of commutative rings, J. Algebra. 320 (7) (2008), 29172933.

[15] M. Ye and T. Wu, Co-maximal ideal graphs of commutative rings, J. Algebra Appl. 11 (6) (2012), Article ID. 1250114 (14 pages).

\section{Subramanian Visweswaran}

Department of Mathematics, Saurashtra University,

Rajkot, India.

S_visweswaran2006@yahoo.co.in

Jaydeep Parejiya

Department of Mathematics,

Saurashtra University,

Rajkot, India.

parejiyajay@gmail.com 